J Urol Nephrol

November 2015 Vol.:2, Issue:2

(c) All rights are reserved by Ekeke et al.

\section{Urological Complications of Obstetrics and Gynaecology Surgeries in a Developing Country}

Keywords: Urological complications; Obstetrics and gynaecological surgery; UPTH

\author{
Abstract \\ Background: Urological complications of obstetrics and \\ gynaecology surgeries are associated with significant morbidity and \\ occasional mortality. Certain factors affect the prevalence and \\ outcome of treatment
}

Aim: To evaluate the risk factors and outcome of treatment of urological complications of obstetrics and gynaecology practice.

Method: This is a retrospectively study of patients who had obstetrics and gynaecology surgeries in the University of Port Harcour Teaching Hospital (UPTH), Port Harcourt between 2009 and 2014. Patients with urological complications were identified. Data on age, clinical presentations, investigations, treatments and outcome of treatment were extracted and analysed using SPSS 20.0

Results: During this period, 8,270 obstetrics and gynaecology surgeries were performed; Twenty five patients $(0.31 \%)$ had urological complications. The mean age was 38.4 years (range $24-62$ years). The commonest initial diagnosis was uterine fibroids in 9 patients followed by uterine rupture in 8 patients. Total abdominal hysterectomy (12 patients) was the commonest surgery. Twenty-one patients had ureteric injury. Seven patients had bladder injury; one patient had injury to the bladder and both ureters. Eight injuries were identified intra-operatively. Ureteric re-implantation was the commonest repair. Twenty two patients (88\%) had satisfactory outcome, one patient (4\%) died due to chronic renal failure while two patients (8\%) had sepsis.

Conclusion: We conclude that conditions that distort pelvic anatomy and emergencies were the major risk factors. Early operative repairs achieved satisfactory results. Early detection and treatmen of urological complications of obstetrics and gynaecology surgeries is important. Good knowledge of anatomy of urogenital system and meticulous surgical technique by the surgeon will reduce the incidence of complications.

\section{Introduction}

The female genital and urinary tracts are anatomically closely related. Therefore, the potential for injury to the urinary system must always be considered when operating on the genital system [1]. Urinary tract injuries are known complications of obstetrics and gynaecology surgeries [2]. Bladder injuries are the most frequent urologic injury inadvertently caused by surgeons [1]. Ureteric injuries are often not recognized immediately and have the potential to be life-threatening. If not treated these injuries may result in permanent kidney damage or removal of a kidney [3].

The incidence of these complications varies worldwide [4-6]. Obstetrics injuries occur mainly in grand multiparous women [4]. The commonest procedure that results in injury is hysterectomy $[4,7,8]$. Radical hysterectomy accounted for 50 percent, 40 percent
Journal of

Urology \& Nephrology

\section{Onyeanunam Ngozi Ekeke*, Ezekiel Olatunde Amusan and Ndubuisi Eke}

Department of Surgery, University of Port Harcourt Teaching Hospital, Port Harcourt, Nigeria

\section{Address for Correspondence}

Onyeanunam N. Ekeke, Department of Surgery, University of Port Harcourt Teaching Hospital, P.O. Box 10697, Port Harcourt, Nigeria, Tel: +234 803338 5956; E-mail: onfekeke@gmail.com

Submission: 31 August, 2015

Accepted: 03 November, 2015

Published: 09 November, 2015

Copyright: () 2015 Ekeke ON et al. This is an open access article distributed under the Creative Commons Attribution License, which permits unrestricted use, distribution, and reproduction in any medium, provided the original work is properly cited.

Reviewed \& Approved by: Dr. Ahmed El-Zawahry, Assistant

Professor of Surgery, Southern Illinois University, USA

from abdominal hysterectomy and less than 5 percent from vaginal hysterectomy [3]. Urological complications may be recognized intraoperatively, some hours, days or weeks after surgery [2,9]. However, about $80 \%$ of injuries are not discovered intraoperatively [3]. They may have more complex complications than those detected intraoperatively $[10,11]$. Post-operative presentations may be loin pain, pyrexia, fistula or non specific signs [12]. Types of injury include ligation, crush, laceration, avulsion, stretch or devascularisation of ureter $[7,8,12]$. Bladder injuries are mainly lacerations and may result in fistulae formation.

The risk factors associated with these injuries have been documented. These include cancer, haemorrhage, endometriosis, adhesions and an enlarge uterus $[12,13]$.

Previous studies in the developing countries highlighted clinical presentations and management of urological complications following obstetrics and gynecology surgery. Late presentation was the most common presentation in these countries [4,7]. A previous study in our centre highlighted iatrogenic urological trauma in both sexes. The ureter and urinary bladder were involved in 35.14\% and 32.43\% respectively [14]. In that study, $38 \%$ of urological complications were due to obstetrics and gynaecological surgeries, of these, $86 \%$ were after hysterectomy. Another study in this centre had focused on urological complication of coitus and urinary tract injury following genital mutilation [15]. The risk factors associated with these injuries in our sub-region and outcome of treatment is yet to be documented. Hence, we have set out in this study to evaluate the various risk factors and treatment outcomes of patients with urological complications following obstetrics and gynaecologic surgery in the University of Port Harcourt Teaching Hospital (UPTH), Port Harcourt, Nigeria.

\section{Materials and Methods}

We retrospectively studied all patients who had obstetrics and gynecology surgeries in UPTH over five years (2009 to 2014). The total 
Citation: Ekeke ON, Amusan EO, Eke N. Urological Complications of Obstetrics and Gynaecology Surgeries in a Developing Country. J Urol Nephrol. 2015;2(2): 6 .

number of patients who had obstetrics and gynaecology surgeries was obtained from the theatre and ward records. Case notes are stored in the medical records department using diagnoses at the hospital's database. Patients with urological complications were identified. Their case notes were retrieved from medical records departments for this study. We evaluated the outcomes of patients treated. This was done internally as part of an audit, so as to improve our quality of care. Since data was retrieved retrospectively with no current contact with the patients, research ethics committee approval was not considered necessary. Patients from another specialist hospital in Port Harcourt, Braithwaite Memorial Specialist Hospital were not included in this study. Therefore some data may be missing especially those that did not involve the urologist in their management.

Urological complications were defined as laceration, transection, rupture or ligation of the urinary tract found during surgery and leakage of urine or contrast media out of urinary tract after surgery that required surgical treatment. Information about patient's demographic characteristics, clinical presentation, investigations, treatment and outcome of treatment were extracted from the case notes.

The following clinical presentation of patients were sought for in the case notes: leakage of urine per vagina, leakage of clear fluid from the operation site, whether the fluid was sent for evaluation and showed elevated creatinine level. Other presentations recorded were presence of abdominal pain, abdominal distension, and prolonged ileus, past abdomino-pelvic surgeries, malignant conditions and duration of urethral catheterization after surgeries. Pre-operative and post-operative radiological imaging films were also retrieved and reviewed.

Results of the full blood count, serum electrolytes, urea and creatinine, urinalysis were checked for each patient. Also recorded were the results of intravenous urography (IVU), voiding cystourethrogram (VCUG) or cystoscopy.

The patients were considered clinically stable and fit for repair if their vital signs, complete blood counts and electrolytes become normal following resuscitation. The outcome of treatment was determined by clinical improvement. The patients were followed up at the outpatient clinics and sometimes using electronic media. Absence of leakage of urine per vagina and from operation serum electrolytes, urea and creatinine were evaluated during follow up visits. Results of post-operative imaging studies were also recorded. The cadre of surgeons was also recorded. The data obtained from the above records were analysed using SPSS version 20.0.0 and presented as charts, tables and figures.

\section{Results}

During the period of study, 8,270 patients had obstetrics and gynaecology surgeries in UPTH. Twenty-five developed urological complications. The overall prevalence of urological complications was $0.31 \%$ while the incidence following obstetrics cases and gynecologic cases were $0.16 \%$ and $0.75 \%$ respectively. The age range of patients was 24-62 years with mean age of $38.4+/-12.6$ years.

Table 1 shows the types of gynaecological and obstetric surgeries that were associated with urological complications. These were uterine fibroid in 11 patients (44\%), uterine rupture in 8 patients (32\%) who had prolonged labour, vesico-vaginal fistula (VVF) in 2 patients $(8 \%)$ while endometrial cancer, adherent placenta, cancer of cervix and ovarian cyst accounted for 1 patient (4\%) each. Most of these patients with large uterine fibroids had lateral displacement of the ureters on intra-venous urography films.

Table 2 shows the main presenting symptoms in the patients with urological complications. The symptoms were mainly leakage of urine at operation sites or per vaginam as well as abdominal distension following gynaecological or an obstetrics operations.

Total abdominal hysterectomy was the most common surgery. This was done in 12 patients. Other surgeries included subtotal hysterectomy (4), ovarian cystectomy (2), Caesarean hysterectomy (3), VVF repair (2) and total abdominal hysterectomy and bilateral salphingo-oophorectomy (2). These were shown in Figure 1. Most of the gynaecological surgeries were elective and were performed by consultants. The obstetric cases were mainly emergencies and were performed mostly by trainee registrars.

Of 25 patients with urological complications, 24 had ureteric injury, either alone or in combination with other injuries. Nine had ligation, 12 had laceration and 3 had total transection of ureter. Seven of 24 had bilateral ureteric injury while 8 and 9 had injury to the right and left ureters respectively. Most of the ureteric injuries occurred in the distal third of the ureters. Minimally invasive techniques were not employed. Stents were only used at the time of open repairs. There were subsequently removed cystoscopically.

Seven patients had bladder injury with one patient having an isolated bladder injury, while 6 had associated ureteric injury. Among these, five bladder injuries each had unilateral ureteric injury and one

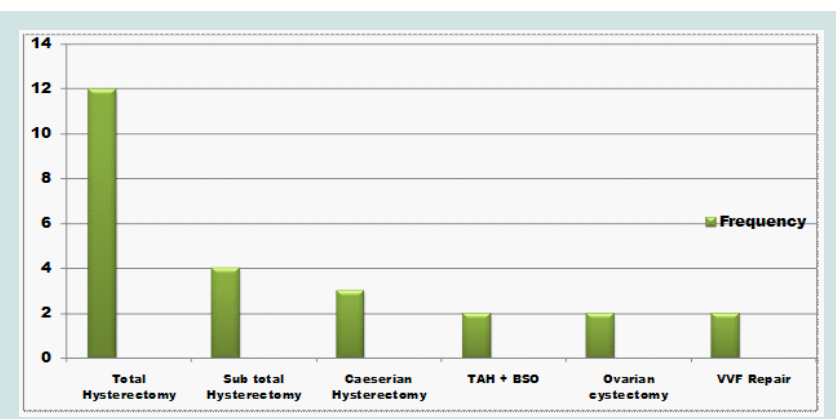

Figure 1: Type of surgery performed in patients with urinary tract injury.

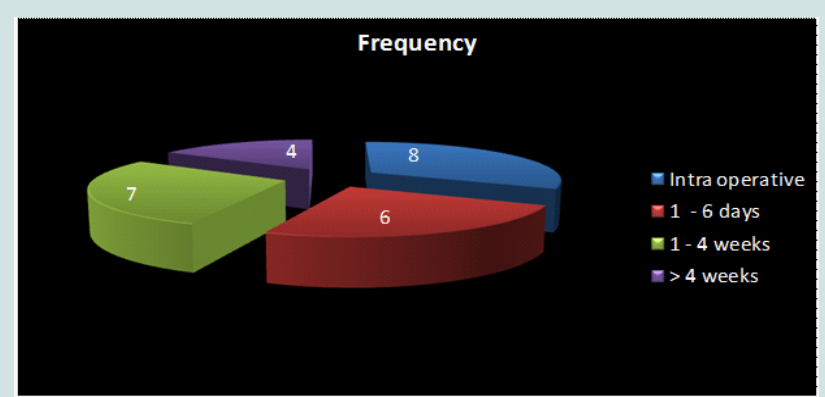

Figure 2: Time of recognition of urinary tract injury. 
Citation: Ekeke ON, Amusan EO, Eke N. Urological Complications of Obstetrics and Gynaecology Surgeries in a Developing Country. J Urol Nephrol. 2015;2(2): 6 .

ISSN: 2380-0585

Table 1: Initial diagnosis leading to obstetric and gynaecologic surgery.

\begin{tabular}{|l|l|c|}
\hline Diagnosis & Frequency & Percent \\
\hline Endometrial cancer & 1 & 4.0 \\
\hline Uterine Fibroids & 11 & 44.0 \\
\hline VVF & 2 & 8.0 \\
\hline Adherent Placenta & 1 & 4.0 \\
\hline Uterine rupture & 8 & 32.0 \\
\hline Ca cervix & 1 & 4.0 \\
\hline Ovarian cyst & 1 & 4.0 \\
\hline Total & 25 & 100 \\
\hline
\end{tabular}

VVF: Vesico-Vaginal Fistula

Table 2: Presenting symptoms of patients with urological complications

\begin{tabular}{|l|l|l|}
\hline Presenting symptoms & Frequency & Percent \\
\hline $\begin{array}{l}\text { Leakage of urine at operation } \\
\text { site }\end{array}$ & 11 & 37.9 \\
\hline Haematuria & 4 & 13.9 \\
\hline Fever & 3 & 10.3 \\
\hline Anuria & 1 & 3.4 \\
\hline Abdominal distension & 3 & 10.3 \\
\hline Haemorhage & 3 & 10.3 \\
\hline Leakage of urine per vagina & 4 & 13.9 \\
\hline Total & $\mathbf{2 9}$ & $\mathbf{1 0 0 . 0}$ \\
\hline
\end{tabular}

sustained bilateral ureteric injuries.

Eight urologic complications were identified intra-operatively and all had ureteric catheter inserted. Of the remaining 17 cases with delayed diagnosis, 6 cases were identified within 6 post-operative days, 7 within 1 and 4 post operative weeks while 4 cases were identified after 4 weeks post-operatively as depicted in Figure 2.

Ureteric repair was the most common procedure. This was done for $10(40 \%)$ patients who had ureteric laceration. Ureteric reimplantation (ureteroneocystostomy) was done for $5(20 \%)$ patients on the left and for $4(16 \%)$ patients on the right. Two (8\%) patients had bilateral ureteroneocystostomy. One (4\%) patient had Boari flap repair and $2(8 \%)$ patients had primary urinary bladder repair as shown in Figure 3. Twenty two patients had satisfactory outcome, 2 patients had sepsis while 1 death was recorded due to chronic renal failure. The patients that had sepsis had immediate repair of ureteric lacerations following a caesarian hysterectomy for ruptured uterus. Their antibiotics were changed on the basis of sensitivity to pseudomonas. They responded to the new antibiotics.

\section{Discussion}

Injury to the urinary tract is a common complication of obstetric and gynaecology surgery [6]. The worldwide incidence is from 0.5 $1.5 \%[16,17]$. Another study in Nigeria reported an incidence of $0.4 \%$ [4]. In this study, the incidence was $0.31 \%$. This is slightly lower than reported incidence. While acknowledging that the incidence is low, this may be due to either good surgical technique by the surgeons or under-reporting of injuries as data available were surgeries in which urologists were involved in the repair. There is no effective system to follow up injuries that do not involve the urologist in their management. Braithwaite Memorial Specialist Hospital also has patients who are managed in their centre with a few of these complications. Some other patients may have been treated in some private hospitals without our knowledge. However, our centre is the main referral centre in our sub region.

Urological complication following obstetric and gynaecology surgery is common in the $4^{\text {th }}$ decade of life [18]. The mean age in a study in Nigeria was 39.4 years [4]. Here, the age range was 24-62 years and the mean age was 38.4 years; this is similar to 34.2 years reported in Middle East [18].

The most common indication for pelvic surgery was uterine fibroid [1]. Seventy-five percent of urinary tract injuries are due to gynaecologic surgery [19]. In this series, the incidence of complications following gynaecologic surgery was $0.75 \%$, with uterine fibroid as the most common indication. Uterine fibroids are prevalent in our environment. They often presented late. Large size of the uterus from fibroids distorts the pelvic anatomy thereby predisposing to injuries. In other studies from Egypt and Jordan, Uterine fibroids did not contribute as much as in this study $[8,18]$.

Other gynaecologic conditions in which injuries included:

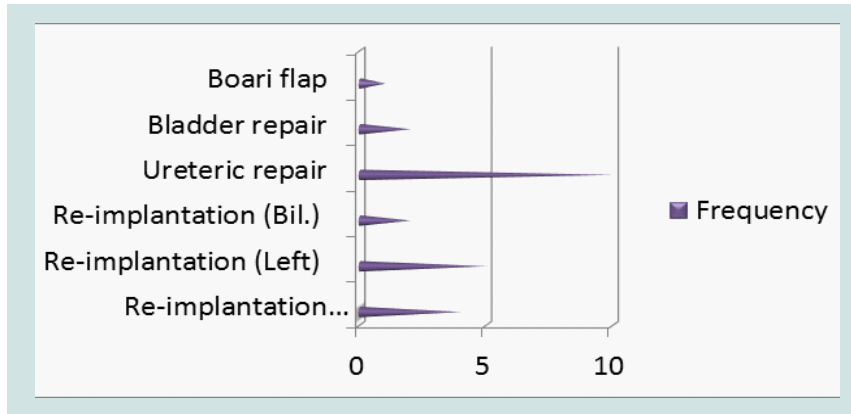

Figure 3: Type of corrective surgery after urinary tract injury.

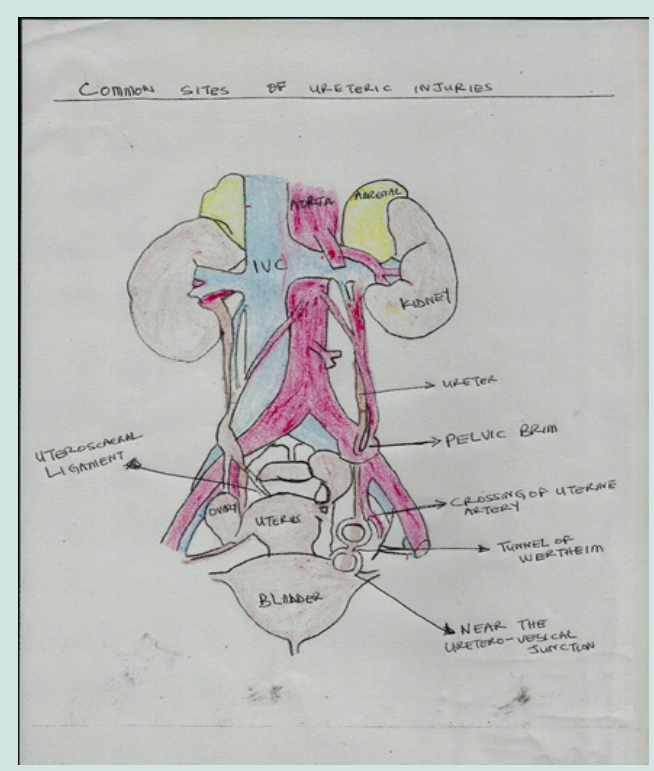

Figure 4: Common sites of ureteric injuries. 
Citation: Ekeke ON, Amusan EO, Eke N. Urological Complications of Obstetrics and Gynaecology Surgeries in a Developing Country. J Urol Nephrol. 2015;2(2): 6 .

ovarian and endometrial cancers as well as endometriosis $[3,5,17,19]$. Though they contributed to the injuries, their contribution in this study was not as much as seen in studies in other centres.

Emergency obstetric conditions may lead to Caesarean sections. The commonest obstetrics conditions recorded in this study were prolonged obstructed labour and ruptured uterus. Though these conditions have been recorded in other studies, they played more roles in this study and featured in this study. Many pregnant women in our environment do not book for antenatal care in hospitals $[5,6]$. They are attended to by unorthodox healers and traditional birth attendants. They only present to our centre with conditions requiring emergency surgeries. The associated massive bleeding and surgeon's attempt to control bleeding often leads to the injuries. Poverty and ignorance have been reported to account for this attitude towards antenatal care [20].

Hysterectomy is the commonest surgery associated with ureteric injuries irrespective of whether it is open or laparoscopic assisted. Abdominal hysterectomy has been associated with high complication rate, though radical hysterectomy had higher complication rate with incidence rate of $0.74 \%$ fistula formation. This has been attributed to wide dissection during the surgery and also the main indication was gynaecologic malignancy [1]. In this study, $84 \%$ of the patients who sustained urological injury had open hysterectomy. No patient had laparoscopic surgery. This technique is yet to be developed in UPTH. Ureteric injuries occur mainly during the stages of ligation of the uterine vessels, dissection of the infundulo-pelvic ligaments as well as ligation of the ovarian vessels [21]. In gynaecological operations the ureter is often injured where it crosses the pelvic brim, uterosacral ligaments, tunnel of Wertheim, uterine artery and near the ureterovesical junction [21]. In pregnancy, because of relaxation of ligaments from hormones, there is a change of relations between the ureter and the ovary - the retroligamentary segment. This location is the most frequently injured site during the ovary mobilization in caesarian hysterectomy [22].

The high rate of injuries following hysterectomy could be explained by the closeness of the bladder and ureter to the uterus. When the anatomy is distorted by a large uterus, as seen in fibroids or uterine malignancies, the possibility of injuries is high. Also, in Caesarean hysterectomy, injuries may occur as a result of severe postpartum hemorrhage. The hurry to control hemorrhage predisposes the patient to injuries in obstetric emergencies. Almost all these women came with unbooked pregnancies resulting in ruptured uteri, thus requiring emergency caesarean section, and often Caesarean hysterectomy. Optimal antenatal care will reduce incidence of these emergency surgeries.

The patient that had cervical cancer had ureteric injury during surgery. Two of the patients with uterine fibroids had hysterectomy due to recurrence. They also had post-operative adhesions which made dissection difficult thereby resulting in ureteric injuries. Malignancies and previous pelvic surgeries may also distort the anatomy and make dissections difficult, thereby leading to injuries to urinary tract during gynecological operations. These conditions, though present our this study, were not as significant as in other studies $[5,6,8,18]$. Pelvic radiation for malignancies has also been reported as risk factors, but this did not play a major role in our study [8]. When distorted anatomy is envisaged, pre-operative radiological investigation and collaboration with the urologists may help to identify ureters and avoid injuries to the ureters during such operations.

The cadre of surgeon who performed the surgery either trainee or consultant may not impact on urological complications [1]. Most obstetric cases are emergencies and associated with haemorrhage were operated on by trainees while most gynaecologic cases were elective and operated on by consultants. We discovered that the incidence of urological complication following obstetric cases was $0.16 \%$. This is lower than incidence from gynaecologic cases which was $0.75 \%$, despite that those most obstetrics cases are done by trainees and gynaecologic cases by consultants.

Injury to the ureter is common during hysterectomy along the pelvic wall lateral to the uterine artery and area of ureterovesical junction $[23,24]$. Therefore, proper understanding of the anatomy and special attention during dissection are important during the surgery [1]. Almost all the patients (96\%) in this study had ureteric injury, either alone or in combination with bladder injury. Ureteric laceration was the most common injury followed by ureteric ligation. This is differs from a study from another region where ureteric ligation was the most common injury [4].

Urinary bladder injury is common during pelvic surgery especially radical vaginal hysterectomy Twenty eight percent had bladder injury. Most bladder injuries were lacerations followed by fistulae. The most common injuries in a report from Egypt were fistulae. Studies from a few centres indicate that bladder injuries were more common than ureteric injuries. This is different from this study where most of the injuries were ureteric $[5,6,18]$. The apparent low prevalence of bladder injuries in this study may be due to under reporting. Most of the bladder injuries recorded in this study were those in which the urologists were involved in their management. No urethral injury was noted in this study unlike in Egypt [17].

Management of ureteric injury either unilateral or bilateral requires insertion of ureteric catheters after repair [22]. In this study, ureteric stents were left in situ for average of six weeks after repair. Injury to both urinary bladder and ureter could occur $[25,26]$. This was reported in $6(24 \%)$ patients in this study. These injuries always followed difficult pelvic surgery in patients with post-op adhesions and malignancies with widespread adhesion in the pelvis as has been seen elsewhere $[1,2]$.

The timing of repair of injury is debatable [27]. Some advocate for immediate repair while others favour delayed repair $[3,28,29]$. The timing of repair in this study varied depending on the time of presentation and clinical state of the patient at presentation. Intraoperative recognition of injury and immediate repair with insertion of ureteric stent and urinary bladder catheter were done for 8 patients in this study as advocated by some urologists [3], this gave better result with few complications. However, most patients in our study were diagnosed post-operatively and had delayed repair, this may have allowed for better tissue plane during dissection, less haemorhage and good outcome as previously advocated [30]. The picture in this study was different from many studies where early recognition and immediate intervention was the predominant pattern $[8,17]$ :

Ureteroneocystostomy was the most common procedure because 
Citation: Ekeke ON, Amusan EO, Eke N. Urological Complications of Obstetrics and Gynaecology Surgeries in a Developing Country. J Urol Nephrol. 2015;2(2): 6 .

most injuries occur in lower third of the ureter. One patient with complete transection of ureter at middle third had boari flap due to short segment of affected ureter and delayed presentation.

Post-operative diagnosis of injury ranged from 2 days to 5 weeks. This is similar to 3-33 days reported elsewhere [31]. Despite the late presentation, most of the patients had satisfactory outcome due to proper evaluation and adequate preparation and optimization before repair of injuries. To optimize the patients, haemodynamic stability, fluid and electrolyte imbalances were corrected. Prophylactic and therapeutic antibiotics: cephalosporins and fluoroquinolones were administered as indicated. Two patients developed severe sepsis, but were successfully treated with antibiotics. Perhaps, delayed repair after ensuring adequate antibiotic cover would have prevented these complications. The patients eventually recovered when the antibiotics were changed to ceftazidime. In our institution, we administer perioperative prophylactic ceftriazone or fluoroquinolones with metronidazole to urology patients. These are usually commenced at induction of anaesthesia. The choice of antibiotics is based on cost, local flora, sensitivity pattern in our environment, side effects and nature of surgery.

The patient who presented with chronic renal failure died despite several sessions of haemodialysis. This patient had bilateral ureteric ligation which was not discovered early. She only presented with severe obstructive nephropathy which did not resolve despite our intervention. Renal failure and overwhelming sepsis have also been documented as cause of death in a similar study in Ghana [32].

This study has some limitations. This was a retrospective study; most patients stopped out-patient clinic attendance when they became symptom free. Therefore, long-term follow up was not possible. We observed that urinary bladder injuries were only reported if there was indication for urologist's intervention. Some patients may have sought for help in some private health facilities. Therefore, bladder injuries were probably under-reported. Our hospital records system is not fully functional. Though there are disease codes, they are not always applied.

\section{Conclusion}

The incidence of urological complications of obstetric and gynaecologic surgery was low, although these contributed to morbidity. Distorted pelvic anatomy from benign and malignant conditions, especially uterine fibroids, as well as obstetric emergencies like ruptured uterus were the major risk factors. Late presentation was predominant. However, the outcome of treatment was satisfactory. Mortality, although low, was avoidable. Improved attitude towards ante-natal care, good knowledge of anatomy of urogenital system and meticulous surgical technique by the surgeon as well as peri-operative collaboration/consultation with a urologist should reduce the rate of complications. There is the need to improve reporting of injuries in the theatres so that accurate data may be available when needed.

\section{References}

1. Lee JS, Choe JH, Lee HS, Seo JT (2012) Urologic complications following obstetric and gynecologic surgery. Korean J Urol 53: 795-799.

2. Obarisiagbon EO, Olagbuji BN, Onuora VC, Oguike TC, Ande AB (2011) latrogenic urological injuries complicating obstetric and gynaecological procedures. Singapore Med J 52: 738-741.
3. Selzman AA, Spirnak JP (1996) latrogenic ureteral injuries: A 20-year experience in treating 165 injuries. J Urol 155: 878-881.

4. Shittu OB, Adeyanju OA, Adebayo SA, Okunlola A, Olayemi OO, et al. (2003) Ureteric injuries arising from obstetric and gynaecological operations at the University College Hospital Ibadan: a 20-year review. Trop J Obstet Gynaecol 20: 32-36.

5. Raut V, Shrivastava A, Nandanwar S, Bhattacharya M (1991) Urological injuries during obstetric and gynaecological surgical procedures. J Postgrad Med 37: 21-23

6. Ozdemir E, Ozturk U, Celen S, Sucak A, Gunel M, et al. (2011) Urinary complications of gynecologic surgery: iatrogenic urinary tract system injuries in obstetrics and gynecology operations. Clin Exp Obstet Gynecol 38: 217220.

7. Aghaji AE, Odoemene C (1999) Ureteric injuries in Enugu, Nigeria. East Afr Med J 76: 184-188.

8. Matani YS, Bani-Hani KE, Bani-Hani IH (2003) Ureteric injuries during obstetric and gynecologic procedures. Saudi Med J 24: 365-368

9. Bentaleb H, Bensouda A, Kabbaj M, Karmouni T, Tazi K, et al. (2007) Prise en charge des traumatismes iatrogènes de l'uretère: A propos de 24 cas. Afr J Urol 13: 219-225

10. Fry DE, Milholen L, Harbrecht PJ (1983) latrogenic ureteral injury: Options in management. Arch Surg 118: 454-457.

11. Nagy V, Valanský L, Milichovský I (1998) Urologic complications of gynecologic surgery and their treatment in our clinical data. Ceska Gynekol 63: 192-195.

12. Drake MJ, Noble JG (1998) Ureteric trauma in gynecologic surgery. Int Urogynecol J 9: 108-117

13. Hwang JH (2012) Urologic complication in laparoscopic radical hysterectomy: meta-analysis of 20 studies. Eur J Cancer 48: 3177-3185.

14. Eke N (2000) latrogenic urological trauma: a 10-year experience from Port Harcourt. West Afr J Med 19: 246-249.

15. Eke N (2002) Urological complications of coitus. BJU Int 89: 273-277.

16. Aronson MP, Bose TM (2002) Urinary tract injury in pelvic surgery. Clin Obstet Gynecol 45: 428-438.

17. Frankman EA, Wang L, Bunker CH, Lowder JL (2010) Lower urinary tract injury in women in the United States, 1979-2006. Am J Obstet Gynecol 202: 495.

18. El-Tabey NA, Ali-El-Dein B, Shaaban AA, El-Kappany HA, Mokhtar AA, et al. (2006) Urological trauma after gynecological and obstetric surgeries. Scand J Urol Nephrol 40: 225-231.

19. Thomson JD (1997) Operative injuries to the ureter: prevention, recognition, and management. In: Rock JA, Thomon JD, (eds). TeLinde's operative gynecology. The d. Philadephia: Lippincott Williams \& Wilkins, pp. 11351174.

20. Fagbamigbe AF, Idemudia ES (2015) Barriers to antenatal care use in Nigeria: evidences from non-users and implications for maternal health programming. BMC Pregnancy Childbirth 15: 95.

21. Badenoch AW (1959) Injuries of the Ureter. Proc R Soc Med 52: 101-108.

22. Cirstoiu M, Munteanu O (2012) Strategies of preventing ureteral iatrogenic injuries in obstetrics-gynecology. J Med Life 5: 277-279.

23. Rao D, Yu H, Zhu H, Duan $P$ (2012) The diagnosis and treatment of iatrogenic ureteral and bladder injury caused by traditional gynaecology and obstetrics operation. Arch Gynecol Obstet 285: 763-765.

24. Liapis A, Bakas P, Giannopoulos V, Creatsas G (2001) Ureteral injuries during gynecological surgery. Int Urogynecol J Pelvic Floor Dysfunct 12: 391 393.

25. Cormio L, Ruutu M, Traficante A, Battaglia M, Selvaggi FP (1993) Management of bilateral ureteric injuries after gynaecological and obstetric procedures. Int Urol Nephrol 25: 551-555. 
Citation: Ekeke ON, Amusan EO, Eke N. Urological Complications of Obstetrics and Gynaecology Surgeries in a Developing Country. J Urol Nephrol. 2015;2(2): 6 .

ISSN: 2380-0585

26. Rafique M, Arif $\mathrm{MH}$ (2002) Management of iatrogenic ureteric injuries associated with gynecological surgery. Int Urol Nephrol 34: 31-35.

27. Soong Y, Lim PH (1997) Urological injuries in gynaecological practice--when is the optimal time for repair? Singapore Med J 38: 475-478.

28. Papanikolaou A, Tsolakidis D, Theodoulidis V, loannidis E, Vatopoulou A et al. (2013) Surgery for ureteral repair after gynaecological procedures: a single tertiary centre experience. Arch Gynecol Obstet 287: 947-950.

29. Badenoch DF, Tiptaft RC, Thakar DR, Fowler CG, Blandy JP (1987) Early repair of accidental injury to the ureter or bladder following gynaecological surgery. Br J Urol 59: 516-518.
30. Moriel EZ, Meirow D, Zilberman M, Farkas A (1993) Experience with the immediate treatment of iatrogenic bladder injuries and the repair of complex vesico-vaginal fistulae by the transvesical approach. Arch Gynecol Obstet 253: $127-130$.

31. Oh BR, Kwon DD, Park KS, Ryu SB, Park YI, et al. (2000) Late presentation of ureteral injury after laparoscopic surgery. Obstet Gynecol 95: 337-339.

32. Mensah JE, Klufio GO, Ahiaku F, Osafo C, Gepi-Attee S (2008) Delayed recognition of bilateral ureteral injury after gynaecological surgery. Ghana Med J 42: 133-136. 